\title{
Another characterisation of planar graphs
}

\author{
C. H. C. Little \\ Massey University, Palmerston North, New Zealand \\ C.Little@massey.ac.nz \\ G. Sanjith* \\ Indian Institute of Technology Madras, Chennai, India \\ theemergingmind@yahoo.co.in \\ Submitted: Sep 13, 2009; Accepted: Mar 1, 2010; Published: Mar 8, 2010 \\ Mathematics Subject Classification: 05C10
}

\begin{abstract}
A new characterisation of planar graphs is presented. It concerns the structure of the cocycle space of a graph, and is motivated by consideration of the dual of an elementary property enjoyed by sets of circuits in any graph.
\end{abstract}

\section{Introduction}

Several characterisations of planar graphs are known. (See [1-20].) We present a new one based on the structure of the cocycle space of a graph.

Let $G$ be a graph with vertex set $V G$ and edge set $E G$. If $S$ and $T$ are disjoint sets of vertices, we denote by $[S, T]$ the set of edges with one end in $S$ and the other in $T$. For any $S \subseteq V G$ we write $\bar{S}=V G-S$, and we define $\partial S=[S, \bar{S}]$. This set is called a cocycle, the cocycle determined by $S$ (or $\bar{S}$ ). A bond is a minimal non-empty cocycle. Thus a non-empty cocycle $\partial S$ in a connected graph $G$ is a bond if and only if $G[S]$ and $G[\bar{S}]$ are both connected. An isthmus is the unique member of a bond of cardinality 1 .

Let $A$ and $B$ be distinct bonds in $G$. We say that two distinct edges of $B-A$ are bound (to each other) by $A$ with respect to $B$ if they join vertices in the same two components of $G-(A \cup B)$.

Now let $A_{1}, A_{2}, A_{3}, B$ be distinct bonds in $G$, and let $a \in B-\left(A_{1} \cup A_{2} \cup A_{3}\right)$. For each $i \in\{1,2,3\}$ let $S_{i}$ be the set of edges bound to $a$ by $A_{i}$ with respect to $B$. We say that $a$ is tied by $\left\{A_{1}, A_{2}, A_{3}\right\}$ with respect to $B$ if the following conditions hold:

\footnotetext{
*Supported by KVPY
} 


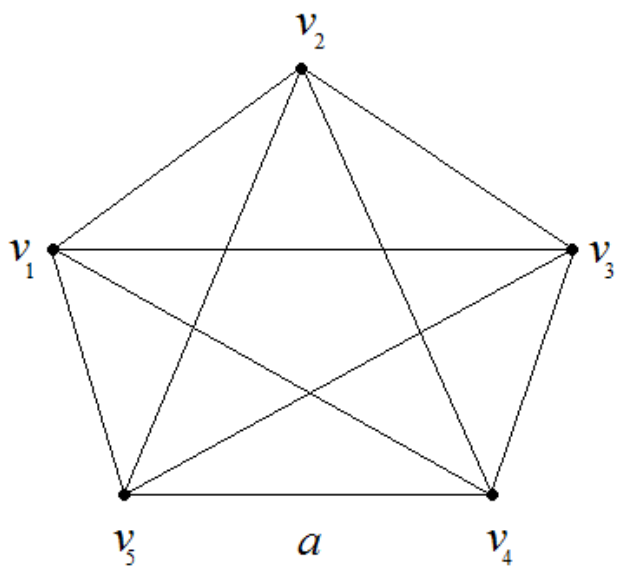

Figure 1: $K_{5}$

1. for each $i$ there is a component of $G-\left(A_{i} \cup B\right)$ that contains an end of $a$ and an end of an edge of $A_{i} \cap B$;

2. each of $S_{1}, S_{2}, S_{3}$ contains an edge that is in neither of the other two.

Edge $a$ is tied if there exist bonds $A_{1}, A_{2}, A_{3}, B$ such that $a$ is tied by $\left\{A_{1}, A_{2}, A_{3}\right\}$ with respect to $B$. The aim of this paper is to prove the following theorem.

Theorem 1.1 A graph is non-planar if and only if it has a tied edge.

We define a circuit in a graph as the edge set of a minimal non-empty connected subgraph in which no edge is an isthmus. In its dual form, the condition stated in Theorem 1.1 is close in spirit to a theorem of Tutte [17] which asserts that a 3-connected graph is planar if and only if every edge is contained in just two induced non-separating circuits, but our result is motivated by consideration of the dual of an elementary observation about the circuits of any graph. (See Theorem 3.1 for the details.) It is similar in flavour to the dual of Fournier's characterisation [7]. However it differs from this dual, which requires three bonds that have at least an edge in common.

\section{Every non-planar graph has a tied edge}

We begin by showing that every edge of $K_{5}$ and $K_{3,3}$ is tied.

Lemma 2.1 Every edge of $K_{5}$ is tied. 


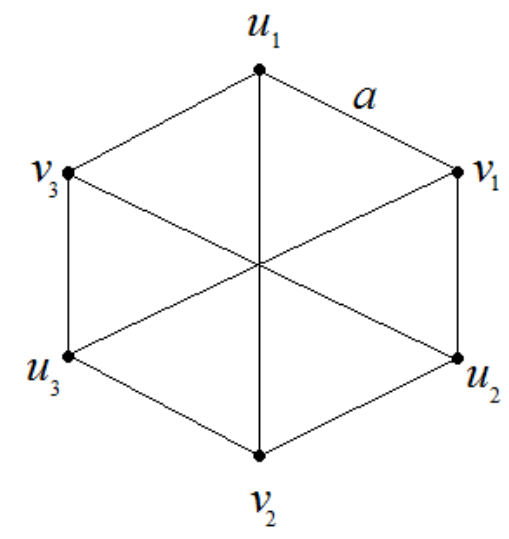

Figure 2: $K_{3,3}$

Proof: Let $V K_{5}=\left\{v_{1}, v_{2}, v_{3}, v_{4}, v_{5}\right\}$, and for each $i$ and $j \neq i$ let $e_{i j}$ be the edge joining $v_{i}$ to $v_{j}$. (See Figure 1.) Without loss of generality, let $a=e_{45}$. Let $Y=\left\{v_{5}\right\}$ and $X_{i}=\left\{v_{i}, v_{4}, v_{5}\right\}$ for each $i$. Define $B=\partial Y$ and $A_{i}=\partial X_{i}$ for each $i$. Then $X_{i} \cap Y=\left\{v_{5}\right\}$ and $X_{i} \cap \bar{Y}=\left\{v_{i}, v_{4}\right\}$ for each $i$. Thus $a \in \partial\left(X_{i} \cap Y\right)=\partial\left\{v_{5}\right\}$ for each $i, e_{25} \in \partial\left\{v_{5}\right\} \cap A_{1} \cap B$ and $e_{15} \in \partial\left\{v_{5}\right\} \cap A_{2} \cap A_{3} \cap B$. We conclude that, for each $i, G\left[\left\{v_{5}\right\}\right]$ is a component of $G-\left(A_{i} \cup B\right)$ containing an end of $a$ and of an edge of $A_{i} \cap B$. Moreover $S_{i}=\left\{e_{i 5}\right\}$ for each $i$. It follows that $a$ is tied by $\left\{A_{1}, A_{2}, A_{3}\right\}$ with respect to $B$.

Lemma 2.2 Every edge of $K_{3,3}$ is tied.

Proof: Let $V K_{3,3}=\left\{u_{1}, u_{2}, u_{3}, v_{1}, v_{2}, v_{3}\right\}$, where each of $u_{1}, u_{2}, u_{3}$ is adjacent to each of $v_{1}, v_{2}, v_{3}$, and for each $i$ and $j$ let $e_{i j}$ be the edge joining $u_{i}$ to $v_{j}$. (See Figure 2.) Let $a=e_{11}$, without losing generality. Let $Y=\left\{u_{1}, v_{2}\right\}, X_{1}=\left\{u_{1}, v_{1}, u_{2}, v_{3}\right\}, X_{2}=$ $\left\{u_{1}, v_{1}, u_{2}, v_{2}\right\}$ and $X_{3}=\left\{u_{1}, v_{1}, v_{2}, u_{3}\right\}$, and define $B=\partial Y$ and $A_{i}=\partial X_{i}$ for each $i$. Then $X_{1} \cap Y=\left\{u_{1}\right\}, X_{1} \cap \bar{Y}=\left\{v_{1}, u_{2}, v_{3}\right\}, X_{2} \cap Y=X_{3} \cap Y=\left\{u_{1}, v_{2}\right\}, X_{2} \cap \bar{Y}=\left\{v_{1}, u_{2}\right\}$ and $X_{3} \cap \bar{Y}=\left\{v_{1}, u_{3}\right\}$. Thus $a \in \partial\left(X_{1} \cap \bar{Y}\right), e_{22} \in \partial\left(X_{1} \cap \bar{Y}\right) \cap A_{1} \cap B, a \in \partial\left(X_{2} \cap Y\right)=\partial\left(X_{3} \cap Y\right)$ and $e_{13} \in \partial\left(X_{2} \cap Y\right) \cap A_{2} \cap A_{3} \cap B$, and so, for each $i$, there is a component $L_{i}$ of $G-\left(A_{i} \cup B\right)$ such that $a \in \partial V L_{i}$ and $\partial V L_{i} \cap A_{i} \cap B \neq \emptyset$. Moreover $S_{1}=\left\{e_{13}\right\}, S_{2}=\left\{e_{22}\right\}$ and $S_{3}=\left\{e_{32}\right\}$. We conclude that $a$ is tied.

We complete the proof that every non-planar graph has a tied edge by applying the following characterisation of planar graphs, due to Wagner [17].

Theorem 2.3 A graph is non-planar if and only if it contains a subgraph contractible to $K_{5}$ or $K_{3,3}$.

In order to reverse the process of contracting an edge, we need the concept of splitting a vertex. Let $e$ be an edge joining distinct vertices $v_{1}$ and $v_{2}$ in a graph $G$. Let $G_{e}$ be 
the graph obtained from $G-\{e\}$ by identifying $v_{1}$ and $v_{2}$ to form a single vertex $v$. Then $G_{e}$ is said to be obtained from $G$ by contracting $e$, and the process of forming $G_{e}$ from $G$ is called a contraction. The graph $G$ is said to be contractible to a graph $H$ if $H$ can be obtained from $G$ by a sequence of contractions. On the other hand, $G$ is said to be obtained from $G_{e}$ by splitting $v$ into $v_{1}$ and $v_{2}$. Observe that any bond of $G_{e}$ is also a bond of $G$. The next lemma and its corollary follow immediately from this observation.

Lemma 2.4 Let $A=\partial X$ and $B=\partial Y$ be bonds of a graph $G$, where $X$ and $Y$ are subsets of $V G$. Let $a \in B-A$, and let $S$ be the set of edges bound to a by $A$ with respect to $B$. Let $G^{\prime}$ be a graph obtained from $G$ by splitting a vertex $v$ into $v_{1}$ and $v_{2}$. For each $Z \in\{X, Y\}$, define $Z^{\prime}=Z$ if $v \notin Z$, and let $Z^{\prime}=(Z-\{v\}) \cup\left\{v_{1}, v_{2}\right\}$ otherwise. Define $A^{\prime}=\partial X^{\prime}$ and $B^{\prime}=\partial Y^{\prime}$, and let $S^{\prime}$ be the set of edges of $G^{\prime}$ bound to a by $A^{\prime}$ with respect to $B^{\prime}$. Then $S^{\prime}=S$.

Corollary 2.5 If an edge is tied in $G$, then it is tied in $G^{\prime}$.

It remains only to reverse the operation of forming a subgraph of a graph. This operation is effected by a sequence of deletions of edges and isolated vertices. We therefore need to consider the adjunction of an edge to a graph $G$, where the new edge joins either two vertices of $G$ or a vertex of $G$ to a new vertex.

Lemma 2.6 Let $A=\partial X$ and $B=\partial Y$ be bonds of a graph $G$, where $X$ and $Y$ are subsets of $V G$, and suppose that $G[X \cap Y]$ and $G[X \cap \bar{Y}]$ are connected. Let $a \in B-A$, and let $S$ be the set of edges bound to a by $A$ with respect to $B$. Let $G^{\prime}$ be a graph obtained from $G$ by the adjunction of an edge e joining two vertices of $G$ or one vertex of $G$ to a vertex not in $G$. For each $Z \in\{X, Y\}$, define $Z^{\prime}=Z$ if e joins two vertices of $G$. If e joins a vertex $x \in V G$ to a vertex $y \notin V G$, then define $Z^{\prime}=Z \cup\{y\}$ if $x \in Z$ and $Z^{\prime}=Z$ otherwise. Define $A^{\prime}=\partial X^{\prime}$ and $B^{\prime}=\partial Y^{\prime}$, and let $S^{\prime}$ be the set of edges of $G^{\prime}$ bound to a by $A^{\prime}$ with respect to $B^{\prime}$. Then $S^{\prime}=S$ or $S^{\prime}=S \cup\{e\}$.

Proof: If $e \in[X \cap Y, X \cap \bar{Y}]$, then $S^{\prime}=S \cup\{e\}$. In the remaining case, $S^{\prime}=S$ since $G[X \cap Y]$ and $G[X \cap \bar{Y}]$ are connected.

Corollary 2.7 If an edge is tied in $G$, then it is tied in $G^{\prime}$.

Proof: Let $a$ be an edge that is tied in $G$ by a set $\left\{A_{1}, A_{2}, A_{3}\right\}$ of bonds with respect to a bond $B$. There exist sets $Y, X_{1}, X_{2}, X_{3}$ of vertices of $G$ such that $B=\partial Y, A_{i}=\partial X_{i}$ for each $i$ and $a \in\left[X_{i} \cap Y, X_{i} \cap \bar{Y}\right]$ for each $i$. Let $L_{i 1}, L_{i 2}, \ldots, L_{i l_{i}}$ be the components of $G\left[X_{i} \cap Y\right]$, where $a$ is incident on a vertex of $L_{i 1}$. By replacing $X_{i}$ with $X_{i}-\bigcup_{j=2}^{l_{i}} V L_{i j}$ if necessary, we adjust each $A_{i}$ so that $G\left[X_{i} \cap Y\right]$ is connected for each $i$. Similarly we further adjust each $A_{i}$, if necessary, so that $G\left[X_{i} \cap \bar{Y}\right]$ is also connected for each $i$. Then $G^{\prime}\left[X_{i}^{\prime} \cap Y^{\prime}\right]$ and $G^{\prime}\left[X_{i}^{\prime} \cap \overline{Y^{\prime}}\right]$ are connected as well, and at least one of them contains an end of an edge of $A_{i}^{\prime} \cap B^{\prime}$ since at least one of $G\left[X_{i} \cap Y\right]$ and $G\left[X_{i} \cap \bar{Y}\right]$ contains an end of an edge of $A_{i} \cap B$. 
Finally, for each $i$, let $S_{i}$ be the set of edges bound to $a$ by $A_{i}$ with respect to $B$. Each of $S_{1}, S_{2}, S_{3}$ has an edge not in either of the others. Using the lemma with $A$ replaced successively by $A_{1}, A_{2}, A_{3}$, we find that $S_{i}^{\prime} \cap E G=S_{i}$ for each $i$. Hence each of $S_{1}^{\prime}, S_{2}^{\prime}, S_{3}^{\prime}$ has an edge not in either of the others. Therefore $a$ is tied in $G^{\prime}$.

Thus the property of an edge being tied is impervious to splittings of vertices and adjunctions of edges. That every non-planar graph has a tied edge now follows from Theorem 2.3, Lemmas 2.1 and 2.2 and Corollaries 2.5 and 2.7.

\section{$3 \quad$ No planar graph has a tied edge}

Why can't a planar graph have a tied edge? The answer lies in the concept of duality. Let $G$ be a planar graph and contemplate its dual $G^{*}$ with respect to a specific embedding of $G$ in the plane. Thus $E G^{*}=E G$ and the vertices of $G^{*}$ are the faces of the embedding of $G-R$ obtained by deleting the set $R$ of isthmuses from the embedding of $G$. Any line segment representing an edge $e$ of $G$ that is not an isthmus separates two distinct faces, and $e$ is incident on those faces in $G^{*}$. If on the other hand $e$ is an isthmus of $G$, then the interior of the line segment representing $e$ is drawn in the interior of a face of the embedding of $G-R$, and $e$ is a loop incident on that face in $G^{*}$. Note that $G^{*}$ is necessarily connected. Each bond of $G$ is a circuit of $G^{*}$, and each circuit of $G$ is a bond of $G^{*}$. If a theorem holds for a given set $T$ of edges of $G$, then the dual of that theorem holds for $T$ in $G^{*}$.

Let $B$ be a circuit in a graph $G$ (not necessarily planar) and let $A$ be another circuit that meets $B$. An $A$-arc of $B$ is a minimal subset $S$ of $B-A$ such that $S \cup A$ includes a circuit distinct from $A$. Thus it is a path included in $B-A$, of minimal length, that joins distinct vertices of $V A \cap V B$. (If $C$ is a circuit in $G$, then we write $V C=V G[C]$.)

Now fix an edge $a \in B-A$. This edge belongs to a unique $A$-arc of $B$. This $A$-arc is called the principal $A$-arc $P(A)$ of $B$ (with respect to $a$ ). The edges of $B-P(A)$ are said to be subtended by $A$ (with respect to $a$ ). These edges form a path included in $B-\{a\}$. We state the following obvious result as a theorem for the sake of emphasis.

Theorem 3.1 Let a be an edge of a circuit $B$ in a graph $G$. Let $A_{1}, A_{2}, A_{3}$ be circuits in $G$ that meet $B$ but do not contain a. Suppose that there is an edge subtended by $A_{1}$ and $A_{3}$ but not $A_{2}$ and an edge subtended by $A_{2}$ and $A_{3}$ but not $A_{1}$. Then any edge subtended by $A_{1}$ and $A_{2}$ is also subtended by $A_{3}$.

Corollary 3.2 Let a be an edge of a circuit $B$ in a graph $G$. Let $A_{1}, A_{2}, A_{3}$ be circuits in $G$ that meet $B$ but do not contain a. Then at least one of $P\left(A_{1}\right), P\left(A_{2}\right), P\left(A_{3}\right)$ is a subset of the union of the others.

We now resuscitate the assumption that $G$ be planar and return to the consideration of $G^{*}$, a graph in which $A_{1}, A_{2}, A_{3}, B$ are bonds. Let $A$ be any circuit of $G$ that meets $B$ but does not contain $a$. Then $A$ is also a bond of $G^{*}$. Let $A=\partial X$ and $B=\partial Y$ for 
some subsets $X$ and $Y$ of $V G^{*}$. Thus $G^{*}[X], G^{*}[\bar{X}], G^{*}[Y], G^{*}[\bar{Y}]$ are all connected. Since $a \in B-A$, we may assume without loss of generality that $a$ joins vertices in $G^{*}[X \cap Y]$ and $G^{*}[X \cap \bar{Y}]$. Let $L$ and $M$ be the components of $G^{*}[X \cap Y]$ and $G^{*}[X \cap \bar{Y}]$, respectively, that contain an end of $a$.

Lemma 3.3 Cocycles $\partial V L$ and $\partial V M$ are bonds of $G^{*}$.

Proof: We shall show only that $\partial V L$ is a bond of $G^{*}$, as the proof that $\partial V M$ is a bond is similar. For this purpose it is enough to show that $G^{*}-V L$ is connected.

Suppose first that $\bar{X} \cap Y=\emptyset$. As $G^{*}[Y]$ is connected, so is $G^{*}[X \cap Y]$, so that $L$ is its only component. Hence $G^{*}-V L=G^{*}[\bar{Y}]$, which is also connected.

We may therefore suppose that $\bar{X} \cap Y \neq \emptyset$. If also $\bar{X} \cap \bar{Y} \neq \emptyset$, then $G^{*}-(X \cap Y)$ is connected since $G^{*}[\bar{X}]$ and $G^{*}[\bar{Y}]$ are connected. If on the other hand $\bar{X} \cap \bar{Y}=\emptyset$, then $G^{*}[\bar{X} \cap Y]$ and $G^{*}[X \cap \bar{Y}]$ are connected for the same reason, so that $M$ is the only component of the latter graph. Once again $G^{*}-(X \cap Y)$ is connected, for $[V M, \bar{X} \cap Y] \neq \emptyset$ since $A$ and $B$ meet.

Thus $G^{*}-(X \cap Y)$ is connected in any case. As $G^{*}[Y]$ is connected, any component of $G^{*}[X \cap Y]$ must have a vertex that is adjacent to some vertex of $G^{*}[\bar{X} \cap Y]$. Therefore $G^{*}-V L$ is connected.

Bonds $\partial V L$ and $\partial V M$ are distinct from $A$ as $a \in(\partial V L \cap \partial V M)-A$. Moreover if $X \cap Y=V L$ then $[V L, V M]$ is a subset of $B-A$ such that $[V L, V M] \cup A$ includes the bond $\partial V M \neq A$. In fact it is a minimal subset $S$ of $B-A$ such that $S \cup A$ includes a bond of $G^{*}$ distinct from $A$. As $a \in[V L, V M]$, it follows that $[V L, V M]=P(A)$. We therefore perceive that if $X \cap Y=V L$ then $P(A)$ consists of $a$ and the edges that are bound to $a$ by $A$ with respect to $B$. By a similar argument we find that the same conclusion holds if $X \cap \bar{Y}=V M$.

We now apply these observations to the bonds $A_{1}, A_{2}, A_{3}$ that meet $B$ but do not contain $a$. Suppose that, for each $i \in\{1,2,3\}$, there is a component $L_{i}$ of $G-\left(A_{i} \cup B\right)$ that contains an end of $a$ and an end of an edge of $A_{i} \cap B$. Let $S_{i}$ be the set of edges bound to $a$ by $A_{i}$ with respect to $B$. Let $A_{i}=\partial X_{i}$. Adjusting $A_{i}$ if necessary, we may suppose, for each $i$, that $X_{i} \cap Y=V L_{i}$ or $X_{i} \cap \bar{Y}=V L_{i}$. Then Corollary 3.2 shows that at least one of $S_{1}, S_{2}, S_{3}$ is included in the union of the others. In other words, $\left\{A_{1}, A_{2}, A_{3}\right\}$ does not tie $a$ with respect to $B$. As $G^{*}$ may be any connected planar graph, no connected planar graph has an edge $a$ and bonds $A_{1}, A_{2}, A_{3}, B$ such that $\left\{A_{1}, A_{2}, A_{3}\right\}$ ties $a$ with respect to $B$. By applying this result to each component of a planar graph, we deduce that no planar graph can have a tied edge. 


\section{References}

[1] D. Archdeacon, C. P. Bonnington, C. H. C. Little, "An algebraic characterization of planar graphs", J. Graph Theory 19 (1995), 237-50.

[2] D. Archdeacon, J. Širáň, "Characterizing planarity using theta graphs", J. Graph Theory 27 (1998), 17-20.

[3] A. A. Chernyak, "Little's conjecture on planar graphs", Izv. Akad. Nauk BSSR Ser. Fiz. Mat. 2 (1980), 41-5 [Russian].

[4] Y. Colin de Verdière, "On a new graph invariant and a criterion for planarity", in "Graph Structure Theory" (N. Robertson, P. Seymour, ed.), Contemp. Math. 147 (1993), Amer. Math. Soc., Providence, 137-47.

[5] H. de Fraysseix, P. Rosenstiehl, "A depth-first-search characterization of planarity", Discrete Math. 13 (1982), 75-80.

[6] H. de Fraysseix, P. Rosenstiehl, "A characterization of planar graphs by Trémaux orders", Combinatorica 5 (1985), 127-35.

[7] J. C. Fournier, "Une relation de séparation entre cocircuits d'un matroide", J. Comb. Theory B16 (1974), 181-90.

[8] F. Jaeger, "Interval matroids and graphs", Discrete Math. 27 (1979), 331-6.

[9] J. Keir, R. B. Richter, "Walks through every edge twice II", J. Graph Theory 21 (1996), 301-10.

[10] K. Kuratowski, "Sur le problème des courbes gauches en topologie", Fund. Math. 15 (1930), 271-83.

[11] C. H. C. Little, D. A. Holton, "Rings of bonds in graphs", J. Comb. Theory B33 (1982), 1-6.

[12] C. H. C. Little, D. A. Holton, "No graph has a maximal 3-ring of bonds", J. Comb. Theory B38 (1985), 139-42.

[13] S. Mac Lane, "A combinatorial condition for planar graphs", Fund. Math. 28 (1937), $22-32$.

[14] W. Schnyder, "Planar graphs and poset dimension", Order 5 (1989), 323-43.

[15] C. Thomassen, "Planarity and duality of finite and infinite graphs", J. Comb. Theory B29 (1980), 244-71.

[16] W. T. Tutte, "Matroids and graphs", Trans. Amer. Math. Soc. 82 (1956), 99-116.

[17] W. T. Tutte, "How to draw a graph", Proc, London Math. Soc. 13 (1963), 743-68.

[18] K. Wagner, "Über eine Eigenschaft der ebenen Komplexe", Math. Ann. 114 (1937), 570-90.

[19] H. Whitney, "Planar graphs", Fund. Math. 21 (1933), 73-84.

[20] S. G. Williamson, "Canonical forms for cycles in bridge graphs", Lin. Multilin. Algebra 34 (1993), 301-41. 